\title{
RANDOMNESS, LOWNESS AND DEGREES
}

\author{
GEORGE BARMPALIAS, ANDREW E. M. LEWIS, AND MARIYA SOSKOVA
}

\begin{abstract}
We say that $A \leq_{L R} B$ if every $B$-random number is $A$-random. Intuitively this means that if oracle $A$ can identify some patterns on some real $\gamma$, oracle $B$ can also find patterns on $\gamma$. In other words, $B$ is at least as good as $A$ for this purpose. We study the structure of the $L R$ degrees globally and locally (i.e., restricted to the computably enumerable degrees) and their relationship with the Turing degrees. Among other results we show that whenever $\alpha$ is not $G L_{2}$ the $L R$ degree of $\alpha$ bounds $2^{\aleph_{0}}$ degrees (so that, in particular, there exist $L R$ degrees with uncountably many predecessors) and we give sample results which demonstrate how various techniques from the theory of the c.e. degrees can be used to prove results about the c.e. $L R$ degrees.
\end{abstract}

\$1. Introduction. One of the goals of this work is to present a uniform approach to studying the $L R$ degrees, both globally and locally. So far the known results about this degree structure have mostly been scattered and in papers dealing with a wider range of themes in algorithmic randomness (see for example [15]). An exception is Simpson's recent paper [20] which deals with themes like almost everywhere domination which are very closely related to the $L R$ degrees. Also, a number of results in this area have been proved via a mix of frameworks like martingales, prefixfree complexity and Martin-Löf tests, with more than one framework sometimes appearing in the same proof (see $[15,14])$. In contrast, we present proofs of new and old results using only the Martin-Löf approach, i.e., $\Sigma_{1}^{0}$ classes and (in the relativised case) c.e. operators. We work in the Cantor space $2^{\omega}$ with the usual topology generated by the basic open intervals

$$
[\sigma]=\left\{\beta \mid \beta \in 2^{\omega} \wedge \sigma \subseteq \beta\right\}
$$

(where $\sigma$ is a finite binary string and $\sigma \subseteq \beta$ denotes that $\sigma$ is a prefix of $\beta$ ) and the Lebesgue measure generated by $\mu([\sigma])=2^{-|\sigma|}$. We systematically confuse sets of finite strings $U$ with the class of reals which extend some string in $U$. Thus

- we write $\mu(U)$ for the measure of the corresponding class of reals

- all subset relations $U \subset V$ where $U, V$ are sets of strings actually refer to the corresponding classes of reals

Received December 30, 2006.

Barmpalias was supported by EPSRC Research Grant No. EP/C001389/1 and would also like to thank Steve Simpson for a number of stimulating discussions, as well as Doug Cenzer for his hospitality during a visit to the University of Florida, September-November 2006. Lewis was supported by MarieCurie Fellowship No. MEIF-CT-2005-023657. Soskova was supported by the Marie Curie Early Training grant MATHLOGAPS (MEST-CT-2004-504029). All authors were partially supported by the NSFC Grand International Joint Project, No. 60310213, New Directions in the Theory and Applications of Models of Computation. 
- Boolean operations on sets of strings actually refer to the same operations on the corresponding classes of reals.

In Section 2 we review the basic definition of an oracle Martin-Löf test and make the simple observation that there exists a universal test with certain nice properties that will later be useful. It is worth noting that not all universal (even unrelativised) Martin-Löf tests have the same properties and for some arguments it is convenient to assume that we hold a special test. This is not new-see for example [11] where a property of the test derived by its construction (and not the general definition) is used to show that random sets are effectively immune. We also briefly survey a few useful known results on Martin-Löf randomness.

In Section 3 we give the definition of $\leq_{L R}$ and the induced degree structure and mention some known properties. We give a slightly different proof of KjosHansen's characterization of $\leq_{L R}$ in terms of relativised $\Sigma_{1}^{0}$ classes of small measure and establish a connection between any two universal oracle tests. We also present Frank Stephan's proof (delivered after a query of the first author) that non-trivial $\leq_{L R}$-upper cones have measure 0 . In Section 4 we show that there is a continuum of reals which are $L R$-reducible to the halting problem and then extend this argument to show that the same is true of any $\alpha$ which is not $G L_{2}$.

In Section 5 we study the structure of the computably enumerable $L R$ degrees. The main goal here is to show how techniques from the theory of the c.e. Turing degrees can be transferred to the c.e. $L R$ degrees. We deal with two fundamental techniques: Sacks coding and Sacks restraints. First we show that if $A$ has intermediate c.e. Turing degree then the lower cone of c.e. $L R$ degrees below it properly extends the corresponding cone of c.e. Turing degrees. The second example demonstrates the use of Sacks restraints in the $L R$ context and is a splitting theorem for the $L R$ degrees: every c.e. set can be split into two c.e. sets in a non-trivial way with respect to $L R$ equivalence. Both of these results concern the relationship between the $L R$ and the Turing degrees. Section 6 is a note on enumerations of random sets. We show that the relativised arithmetical and Ershov hierarchies are still proper when restricted to the class of random sets. Finally in Section 7 we present an adaptation of Nies' original argument that low for random sets are $\Delta_{2}^{0}$ which is phrased entirely in the framework of Martin-Löf tests and provides some information about universal tests of a certain type.

§2. Oracle Martin-Löf tests. An oracle Martin-Löf test $\left(U_{e}\right)$ is a uniform sequence of oracle machines which output finite binary strings such that if $U_{e}^{\beta}$ is the range of the $e$-th machine with oracle $\beta \in 2^{\omega}$ then for all $\beta \in 2^{\omega}$, e $\in \mathbb{N}$, $\mu\left(U_{e}^{\beta}\right)<2^{-(e+1)}$ and $U_{e}^{\beta} \supseteq U_{e+1}^{\beta}$. A real $\alpha$ is called $\beta$-random if for every oracle Martin-Löf test $\left(U_{e}\right)$ we have $\alpha \notin \cap_{e} U_{e}^{\beta}$. A universal oracle Martin-Löf test is an oracle Martin-Löf test $\left(U_{e}\right)$ such that for every $\alpha, \beta \in 2^{\omega}, \alpha$ is $\beta$-random iff $\alpha \notin \cap_{e} U_{e}^{\beta}$. Given any oracle Martin-Löf test $\left(U_{e}\right)$, each $U_{e}$ can be thought of as a c.e. set of axioms $\langle\tau, \sigma\rangle$. If $\beta \in 2^{\omega}$ then

$$
U_{e}^{\beta}=\left\{\sigma \mid \exists \tau\left(\tau \subset \beta \wedge\langle\tau, \sigma\rangle \in U_{e}\right)\right\}
$$

and for $\rho \in 2^{<\omega}$ we define

$$
U_{e}^{\rho}=\left\{\sigma \mid \exists \tau\left(\tau \subseteq \rho \wedge\langle\tau, \sigma\rangle \in U_{e}\right)\right\} .
$$


There is an analogy between oracle Martin-Löf tests as defined above and Lachlan functionals i.e., Turing functionals viewed as c.e. sets of axioms. This analogy will be exploited in a number of constructions below, especially in the constructions of c.e. $L R$ degrees. The following lemma is easily proved and provides a universal oracle Martin-Löf test with properties which will later be useful.

Lemma 2.1. There is an oracle Martin-Löf test $\left(U_{e}\right)$ such that

- For every oracle Martin-Löf test $\left(V_{e}\right)$, uniformly on its c.e. index we can compute $k \in \mathbb{N}$ such that for every real $\beta$ and all $e, V_{e+k}^{\beta} \subseteq U_{e}^{\beta}$.

- If $\left\langle\tau_{1}, \sigma_{1}\right\rangle,\left\langle\tau_{2}, \sigma_{2}\right\rangle \in U_{e}$ and $\tau_{1} \subseteq \tau_{2}$ then $\sigma_{1} \mid \sigma_{2}$.

- If $\langle\tau, \sigma\rangle \in U_{e}$ then $|\tau|=|\sigma|$ and $\langle\tau, \sigma\rangle \in U_{e}[|\tau|]-U_{e}[|\tau|-1]$.

Proof. Suppose that $\Phi_{i}$ is an effective enumeration of all oracle Martin-Löf tests, so that $\Phi_{i}(e)$ is the $e$-th member of the $i$-th oracle Martin-Löf test. We enumerate a test such that the above conditions are satisfied. Given $e$ we wish to enumerate into the $e$-th member of the test all $\langle\tau, \sigma\rangle$ which belong to $\Phi_{i}(e+i+1)$. This would give an oracle Martin-Löf test $\left(E_{e}\right)$ which satisfies the first condition. To satisfy the second and third conditions as well we do the same but with a modification. If at some stage $s$ we would enumerate $\langle\tau, \sigma\rangle$ into $E_{e}$ according to the construction just described, now we split $\tau$ into a prefix-free finite set $B$ of strings of length $s$ (i.e., $B$ is the set of strings of length $s$ which extend $\tau), \sigma$ into a prefix-free finite set $C$ of strings of length $s$ and replace $\langle\tau, \sigma\rangle$ with the axioms $\left\langle\tau^{\prime}, \sigma^{\prime}\right\rangle$ for all $\tau^{\prime} \in B, \sigma^{\prime} \in C$. We enumerate into $U_{e}$ each string $\left\langle\tau^{\prime}, \sigma^{\prime}\right\rangle$ with $\tau^{\prime} \in B, \sigma^{\prime} \in C$ such that for every $\langle\rho, \zeta\rangle$ currently in $U_{e}, \rho \nsubseteq \tau^{\prime}$ or $\zeta \nsubseteq \sigma^{\prime}$. It is easy to verify that the new test $\left(U_{e}\right)$ is essentially the same as the simpler $\left(E_{e}\right)$ in the sense that for every $\beta \in 2^{\omega}$ and $e \in \mathbb{N}$ the class of reals in $U_{e}^{\beta}$ is the same as the class of reals determined by $E_{e}^{\beta}$.

Corollary 2.2. Let $\left(U_{e}\right)$ be the universal oracle Martin-Löf test of Lemma 2.1 and let $U$ be any member of it. There is a computable function which, given any input $\left\langle\tau, \tau^{\prime}\right\rangle$ such that $\tau \subseteq \tau^{\prime}$, outputs the finite (clopen) set $U^{\tau^{\prime}}-U^{\tau}$.

Proof. This follows from the properties of $\left(U_{e}\right)$ as described in Lemma 2.1. $\dashv$

If $U, V$ are two prefix-free sets of strings it is not hard to show that $U V$ is prefix free and $\mu(U V)=\mu(U) \cdot \mu(V)$, where

$$
U V=\{\sigma \tau \mid \sigma \in U \wedge \tau \in V\}
$$

Hence for any $n, \mu\left(U^{n}\right)=(\mu(U))^{n}$. However if $U$ is not prefix-free this may not hold (it is not hard to find a counterexample). For this reason, wherever we consider such products below we always assume that the underlying sets are prefixfree. It is also worth noting that if $U_{1}, U_{2}$ are two different prefix-free sets of strings representing the same open set of reals, $U_{1}^{2}$ does not necessarily represent the same set of reals as $U_{2}^{2}$ (e.g., consider the case where $U_{1}=\{0\}$ and $U_{2}=\{00,01\}$ ). However this fact will not cause any problems in what follows as we only deal with a single representation for a given open set.

In the following we survey some fundamental known results which are needed in order to understand Bjørn Kjos-Hanssen's characterisation of $\leq_{L R}$ which is presented in Section 3. Every $\Pi_{1}^{0}$ class containing a random real must have positive measure. In order to see this observe that otherwise we would be able to use this $\Pi_{1}^{0}$ class in order to define a Martin-Löf test which contains precisely the members 
of that $\Pi_{1}^{0}$ class, giving a contradiction. This observation clearly relativises to any oracle $B$. This implies that if $\left(V_{e}^{B}\right)$ is a universal Martin-Löf test relative to $B$ then for every $e$ and $\sigma$ either $[\sigma] \subseteq V_{e}^{B}$ or

$$
\mu_{\sigma}\left(V_{e}^{B}\right):=\frac{\mu\left([\sigma] \cap V_{e}^{B}\right)}{2^{-|\sigma|}}<1
$$

(note that the equality above denotes a definition of the leftmost term). In order to see this observe that if $[\sigma] \nsubseteq V_{e}^{B}$ then the reals which extend $\sigma$ and do not belong to $V_{e}^{B}$ are all $B$-random and form a nonempty $\Pi_{1}^{0}$ class relative to $B$. This class must therefore have positive measure. Finally it is useful to know that if $P$ is a $\Pi_{1}^{0}$ class of positive measure, then every random real has a tail in $P$ [11]. Indeed, consider the complement $V$ of $P$ as a prefix-free c.e. set of strings and a random $\beta$. Then $\mu(V)<1$ and there exists a computable function $f$ such that $\left(V^{f(n)}\right)$ is a Martin-Löf test. Thus there exists a least $n$ such that $\beta \notin V^{n}$ and a $\sigma \in V^{n-1}$ such that $\sigma \subset \beta$. This means that the tail of $\beta$ starting from position $|\sigma|$ belongs to $P$. This observation relativises to any oracle.

§3. $L R$ reducibility and degrees. The $L R$ reducibility was introduced in [16].

Definition 1. [16] Let $A \leq_{L R} B$ if every $B$-random real is $A$-random. The induced degree structure is called the $L R$ degrees.

Intuitively this means that if oracle $A$ can identify some patterns on some real $\gamma$, oracle $B$ can also find patterns on $\gamma$. In other words, $B$ is at least as good as $A$ for this purpose. It is not hard to show (especially in view of Theorem 3.2) that $\leq_{L R}$ is $\Sigma_{3}^{0}$ definable and this has been noticed by a number of authors. Being $\Sigma_{3}^{0}$ means that it has some things in common with $\leq_{T}$ (which is also $\Sigma_{3}^{0}$ ) and this can be seen more clearly in Section 5 where techniques from the theory of c.e. Turing degrees are seen to be applicable in the c.e. $L R$ degrees. For more examples of similar $\Sigma_{3}^{0}$ relations see [20]. We point out (after $[16,20])$ that a strict relativization of the notion of low-for-random [12] gives that $A$ is low-for-random relative to $B$ when $A \oplus B \leq_{L R} B$, which is different than $A \leq_{L R} B$. In particular, $\oplus$ does not define join in the $L R$ degrees and it is an open question as to whether any two degrees always have a least upper bound in this structure [16, 20].

LEMma 3.1. [9] If $U_{1}, U_{2}$ are $\Sigma_{1}^{0}(A)$ classes and $U_{1} U_{2} \subseteq V$ where $V$ is a $\Sigma_{1}^{0}(B)$ class of measure less than 1 then at least one of the following holds:

- there exists $V_{1} \in \Sigma_{1}^{0}(B)$ of measure less than 1 such that $U_{1} \subseteq V_{1}$.

- there exists $V_{2} \in \Sigma_{1}^{0}(B)$ of measure less than 1 such that $U_{2} \subseteq V_{2}$.

Hence (by iteration), if $U$ is a $\Sigma_{1}^{0}(A)$ class and $U^{n} \subseteq V$ where $V$ is a $\Sigma_{1}^{0}(B)$ class of measure less than 1 then there exists $V_{1} \in \Sigma_{1}^{0}(B)$ of measure less than 1 such that $U \subseteq V_{1}$.

Proof. It suffices to prove the first clause. First suppose that there is $\sigma$ such that $\mu_{\sigma}(V)<1$ and $\sigma \in U_{1}$. Then define

$$
V_{2}=\{\tau \mid \sigma \tau \in V\}
$$

and note that $U_{2} \subseteq V_{2}$ and $\mu\left(V_{2}\right)<1$. Otherwise let $q$ be a rational such that $\mu(V)<1-q$, define

$$
V_{1}=\left\{\sigma \mid \mu_{\sigma}(V)>1-q\right\}
$$


and note that $U_{1} \subseteq V_{1}$ and $(1-q) \cdot \mu\left(V_{1}\right) \leq \mu(V)$ (so $\mu\left(V_{1}\right)<1$ ).

THEOREM 3.2. [9] For all $A, B \in 2^{\omega}$ the following are equivalent:

- $A \leq_{L R} B$.

- For every $\Sigma_{1}^{0}(A)$ class $T^{A}$ of measure $<1$ there is a $\Sigma_{1}^{0}(B)$ class $V^{B}$ such that $\mu\left(V^{B}\right)<1$ and $T^{A} \subseteq V^{B}$.

- For some member $U^{A}$ of a universal Martin-Löf test relative to A there is $V^{B} \in \Sigma_{1}^{0}(B)$ such that $\mu\left(V^{B}\right)<1$ and $U^{A} \subseteq V^{B}$.

Proof. The following proof is essentially the one presented in [9] but without the use of prefix-free machines. First suppose that $A \leq_{L R} B$, i.e., that every $B$-random is $A$-random. Let $\left(V_{e}^{B}\right)$ be a universal Martin-Löf test relative to $B$ and let $\left(U_{e}\right)$ be the test of Lemma 2.1. Then there must be a string $\sigma$ and $m \in \mathbb{N}$ such that $[\sigma] \nsubseteq V_{0}^{B}$ and

$$
U_{m}^{A} \cap[\sigma] \subseteq V_{0}^{B} \cap[\sigma] .
$$

Indeed, if the negation of this held then we could use it to construct (by finite extensions) a real which belongs to $U_{e}^{A}$ for all $e$ but does not belong to $V_{0}^{B}$; that is, a $B$-random real which is not $A$-random.

Now it is not hard to construct a $\Sigma_{1}^{0}(B)$ class $W^{B}$ such that $\mu\left(W^{B}\right)<1$ and $U_{m}^{A} \subseteq W^{B}$. We just have to enumerate into $W^{B}$ all reals outside the cone $[\sigma]$ plus the reals in $[\sigma] \cap V_{0}^{B}$. Then by (2) we have $U_{m}^{A} \subseteq W^{B}$ and since $\mu_{\sigma}\left(V^{B}\right)<1$ we also have $\mu\left(W^{B}\right)<1$. Now consider a $\Sigma_{1}^{0}(A)$ class $T^{A}$ of measure $<1$ (consider $T^{A}$ as a prefix-free set of strings). Then some subset of the sequence $\left(\left(T^{A}\right)^{k}\right)$ is a Martin-Löf test relative to $A$. By the properties of $\left(U_{e}\right)$ there exists $k$ such that $\left(T^{A}\right)^{k} \subseteq U_{m}^{A}$. Hence $\left(T^{A}\right)^{k} \subseteq W^{B}$ and by Lemma 3.1 this proves the second clause of the claim.

The second clause obviously implies the third clause so we are left to derive the first clause from the third clause. Suppose that $\beta$ is $B$-random and $V^{A} \subseteq T^{B}$ where $V^{A}$ is a member of a universal Martin-Löf test relative to $A, T^{B} \in \Sigma_{1}^{0}(B)$ and $\mu\left(T^{B}\right)<1$. Then the complement of $T^{B}$ contains a tail of $\beta$, so the complement of $V^{A}$ also contains that tail of $\beta$. Since $V^{A}$ belongs to a universal test, $\beta$ is $A$-random.

The following result shows how two universal oracle Martin-Löf tests are related (or how 'similar' they are) and its proof is in the spirit of the proof of Theorem 3.2.

THEOREM 3.3. If $\left(U_{i}\right)$ is an oracle Martin-Löf test, $V$ is a member of a universal oracle Martin-Löf test and $\tau_{0}, \sigma_{0} \in 2^{<\omega}$ such that $\left[\sigma_{0}\right] \nsubseteq \cap_{\gamma \supset \tau_{0}} V^{\gamma}$ then there exist $\tau, \sigma \in 2^{<\omega}, m \in \mathbb{N}$ such that

- $\tau \supset \tau_{0}$ and $\sigma \supset \sigma_{0}$,

- there is $\beta \supset \tau$ such that $[\sigma] \nsubseteq V^{\beta}$,

- for all $\gamma \supset \tau, U_{m}^{\gamma} \cap[\sigma] \subseteq V^{\gamma}$.

Proof. Assuming that the claim does not hold we will construct $\alpha, \delta \in 2^{\omega}$ such that $\alpha \in \cap_{i} U_{i}^{\delta}$ and $\alpha \notin V^{\delta}$, which is clearly a contradiction. Let $\alpha_{0}=\sigma_{0}, \delta_{0}=\tau_{0}$ and at stage $s>0$ pick strings $\sigma \supset \alpha_{s-1}$ and $\tau \supset \delta_{s-1}$ such that

- there is $\gamma \in[\tau]$ such that $[\sigma] \not \subset V^{\gamma}$,

- $[\sigma] \subseteq U_{s}^{\tau}$ 
and set $\alpha_{s}=\sigma, \delta_{s}=\tau$. These strings will exist by the assumptions about $\sigma_{0}, \tau_{0}$ (for the first step) and the assumption that the claim does not hold. Now if $\alpha=\cup_{s} \alpha_{s}$, $\delta=\cup_{s} \delta_{s}$ then $\alpha \in \cap_{i} U_{i}^{\delta}$ and $\alpha \notin V^{\delta}$.

A natural question about reducibilities $\preceq$ on the reals is to determine the measure of upper and lower cones. For the Turing reducibility the lower cones are countable (hence they are null) and the non-trivial upper cones have measure 0 [18]. For $\leq_{L R}$ although lower cones are not always countable (see Section 4) it is not difficult to show that they are null.

THEOREM 3.4. For every $A$ the set $\left\{\beta \mid \beta \leq_{L R} A\right\}$ has measure 0 .

Proof. Given $A$ the $A$-random numbers have measure 1 and so it is enough to show that if $\beta$ is $A$-random then $\beta \not_{L R} A$. But this is obvious since $\beta$ is not $\beta$-random.

For the upper cones it is tempting to think that a version of the majority vote technique which settled the question for $\leq_{T}$ (see [5] for an updated presentation of the argument) would work for $\leq_{L R}$ (especially if one thinks of randomness in terms of betting strategies). However Frank Stephan pointed out (in discussions with the first author) that the answer is most easily given by an application of van Lambalgen's theorem (a simple theorem with many applications) which asserts that $A \oplus B$ is random iff $A$ is random and $B$ is $A$-random.

THEOREM 3.5 (Frank Stephan). If $A$ is random then it is $B$-random for almost all $B \in 2^{\omega}$. Also, any non-trivial upper cone in the $L R$ degrees has measure 0 .

Proof. For the first claim, let $A$ be random. Since the random sets have measure 1 , the measure of sets $B$ such that $A$ is $B$-random equals the measure of random sets $B$ with the same property. By van Lambalgen's theorem (and since $A$ is random) these are the sets $B$ which are $A$-random. But these have measure 1 .

For the second clause it suffices to show that if $A$ is not low for random then the measure of sets $B$ such that every $B$-random real is $A$-random is 0 . If $A$ is not low for random there is a random $R$ which is not $A$-random. Since (by the first clause) $R$ is $B$ random for almost all $B$, the class of $B$-randoms is not contained in the class of $A$-randoms for almost all $B$.

$\S 4$. Uncountable predecessors. In computability theory we are used to structures in which every degree has only countably many predecessors. Below we show that the $L R$ degrees do not have this property ${ }^{1}$ and that, in fact, whenever $\alpha$ is not $G L_{2}$ the degree of $\alpha$ has an uncountable number of predecessors.

Lemma 4.1. Let $U$ be a member of an oracle Martin-Löf test, $n \in \mathbb{N}$ and $\tau_{0} \in 2^{<\omega}$. Then there exists $\tau_{1} \supset \tau_{0}$ such that for all $\tau_{2} \supset \tau_{1}, \mu\left(U^{\tau_{2}}-U^{\tau_{1}}\right)<2^{-n}$.

Proof. Suppose towards a contradiction that the claim of the lemma does not hold. Then there exists $n \in \mathbb{N}$ such that every extension $\tau_{1}$ of $\tau_{0}$ can be extended to $\tau_{2}$ such that $\mu\left(U^{\tau_{2}}-U^{\tau_{1}}\right) \geq 2^{-n}$. We can therefore construct by using a finite number of extensions $\tau_{1}$ such that $\mu\left(U^{\tau_{1}}\right)>2^{-1}$.

In [16] (see [20] for a different proof and more detailed presentation) it was shown that the $L R$ degrees are countable equivalence classes.

\footnotetext{
${ }^{1}$ Joe Miller and Yu Liang have independently announced the existence of an $L R$ degree with uncountably many predecessors.
} 
THeOREM 4.2. In the LR degrees the degree of $\emptyset^{\prime}$ bounds $2^{\aleph_{0}}$ degrees.

Proof. By cardinal arithmetic it is enough to show that the set $\mathscr{B}=\left\{\beta \mid \beta \leq_{L R}\right.$ $\left.\emptyset^{\prime}\right\}$ has cardinality $2^{\aleph_{0}}$. Let $U$ be the second member of the universal oracle MartinLöf test of Lemma 2.1, so that by definition $\mu\left(U^{\beta}\right)<2^{-2}$ for all $\beta \in 2^{\omega}$. It suffices to define a $\emptyset^{\prime}$-computable perfect tree $T$ (as a map from $2^{<\omega}$ to itself which preserves the prefix and incompatibility relations) such that

$$
\mu(A) \leq \frac{1}{2} \text { where } A=\cup_{\tau \in T} U^{\tau} .
$$

Then $|[T]|=2^{\aleph_{0}}$ (where $[T]$ is the set of infinite paths through $T$ ), and for all $\beta \in[T], U^{\beta} \subseteq A$. Since $A$ is $\emptyset^{\prime}$-c.e., we have by Theorem 3.2 that for all $\beta \in[T]$, $\beta \leq_{L R} \emptyset^{\prime}$. We ask that $\mu(A) \leq \frac{1}{2}($ rather than $\mu(A)<1)$ simply in order that figures used should be in line with what will appear in the proof of Theorem 4.3.

It remains to define such a tree $T$ and verify the construction. First find a string $\tau$ such that for any extension $\tau^{\prime}$ of $\tau, \mu\left(U^{\tau^{\prime}}-U^{\tau}\right)<2^{-4}$ and define $T(\emptyset)=\tau$. The existence of such a string is ensured by Lemma 4.1. Note that $\mu\left(U^{T(\emptyset)}\right)<2^{-2}$. Now for each of the one element extensions of $T(\emptyset)$, say $\tau_{i}, i=0,1$ find some extension $\tau_{i}^{\prime} \supseteq \tau_{i}$ such that for any $\tau^{\prime} \supset \tau_{i}^{\prime}$ we have $\mu\left(U^{\tau^{\prime}}-U^{\tau_{i}^{\prime}}\right)<2^{-6}$. Define $T(0)=\tau_{0}^{\prime}, T(1)=\tau_{1}^{\prime}$ and note that $\mu\left(\left(U^{T(0)} \cup U^{T(1)}\right)-U^{T(\emptyset)}\right)<2 \cdot 2^{-4}=2^{-3}$ by the previous step. Continue in the same way so that at the $n$-th stage, where we define $T(\sigma)$ for all $\sigma$ with $|\sigma|=n$, we choose a value $\tau$ for $T(\sigma)$ such that for all $\tau^{\prime} \supset \tau$ we have $\mu\left(U^{\tau^{\prime}}-U^{\tau}\right)<2^{-(2 n+4)}$. Let

$$
C_{n}=\left\{T(\sigma)\left|\sigma \in 2^{<\omega} \wedge\right| \sigma \mid \leq n\right\}
$$

and note that $C_{n} \subseteq C_{n+1}$. Also let

$$
A_{n}=\cup_{\tau \in C_{n}} U^{\tau}
$$

and note that $A_{n} \subseteq A_{n+1}$ and $A=\cup_{n} A_{n}$. By induction, for all $n$

$$
\mu\left(A_{n}\right)<\sum_{i=0}^{n} 2^{i} \cdot 2^{-(2 i+2)}=2^{-2}+2^{-3}+\cdots=\frac{1}{2} .
$$

Note that the factor $2^{i}$ in the above sum comes from the number of strings of level $i$ in $T$ (and where we say that $\tau$ is of level $i$ in $T$ if $\tau=T(\sigma)$ for $\sigma$ of length $i$ ). It remains to show that we can run the construction of $T$ computably in $\emptyset^{\prime}$, but this follows immediately from Corollary 2.2 .

After we proved Theorem 4.2 and since high degrees often resemble $\boldsymbol{O}^{\prime}$, we considered showing that every high $L R$ degree has uncountably many predecessors. Using a combination of highness techniques from $[8,13,19]$ we succeeded in showing that if $A$ is generalized superhigh (i.e., $\left.A^{\prime} \geq_{t t}\left(A \oplus \emptyset^{\prime}\right)^{\prime}\right)$ then $A$ has uncountably many $\leq_{L R}$-predecessors. The following theorem is a stronger result showing that if $A$ is merely $\overline{G L}_{2}$ (i.e., generalized non-low $\left.2, A^{\prime \prime}>_{T}\left(A \oplus \emptyset^{\prime}\right)^{\prime}\right)$ then it has the same property. For other $\overline{G L}_{2}$ constructions we refer the reader to [13].

THeOREM 4.3. If $\alpha$ is $\overline{G L}_{2}$ then in the LR degrees the degree of $\alpha$ bounds $2^{\aleph_{0}}$ degrees.

Proof. The basic idea behind the proof remains the same as in the proof of Theorem 4.2 but now we proceed to define $T$ using only an oracle for $\alpha$ rather than an oracle for $\emptyset^{\prime}$. In order to do so we drop the requirement that $T$ should 
be perfect-we allow that some strings in $T$ have no extensions in $T$-and we $\alpha$ approximate a tree $T^{*} \subseteq T$ which is perfect during the course of the construction. Once again we let $U$ be the second member of the universal oracle Martin-Löf test of Lemma 2.1. We ensure that

$$
\mu(A) \leq \frac{1}{2} \text { where } A=\cup_{\tau \in T} U^{\tau} .
$$

The fact that $T^{*}$ is a subtree of $T$ and is perfect ensures that there exist $2^{\aleph_{0}}$ infinite paths through $T$. To make sure that our approximation to $T^{*}$ converges we will use the fact that $\alpha$ is $\overline{G L}_{2}$.

The construction will be $\alpha$-computable and will enumerate a set of strings $T$ such that (3) holds. Every time we enumerate a string in $T$ we do it in order to extend $T^{*}$, wishing that it can be made perfect in the limit. In order to ensure that (3) held in the proof of Theorem 4.2 we first enumerated one string adding less than $2^{-2}$ to $\mu(A)$, then two strings adding less than $2^{-4}$ each to $\mu(A)$, and then for every $n$ we enumerated $2^{n}$ strings adding less than $2^{-2 n-2}$ each to $\mu(A)$. Now we extend that idea. We use movable markers which take positions on strings in the current version of the tree $T$. At any stage we shall have at most one marker of the form $l_{0}$, at most two markers of the form $l_{1}$, and in general, for any $n$ we have at most $2^{n}$ markers of the form $l_{n}$. At every stage of the construction we ensure that the following condition is satisfied:

For every $n$ there exist at most $2^{n}$ strings in $T$ allocated a marker $l_{n}$.

Each string allocated a marker $l_{n}$ adds less than $2^{-2 n-2}$ to $\mu(A)$ and

all measure in $A$ can be attributed to some string with a marker (i.e.,

for every $\tau \in T$ there exists $\tau^{\prime} \supseteq \tau$ in $T$ which is allocated a marker).

Clearly if we ensure that (4) is satisfied at every stage of the construction we shall have that $\mu(A) \leq \frac{1}{2}$.

Using the fact that $\alpha \in \overline{G L}_{2}$. We shall use the fact that for every function $f \leq_{T} \emptyset^{\prime}$ there exists a function $h \leq_{T} \alpha$ which is not dominated by $f$ (i.e., which is larger than $f$ on an infinite number of arguments). Let the finite binary strings be ordered by length and then from left to right and let $\emptyset$ denote the empty string (at least for the duration of this proof). Given any $\tau \in 2^{<\omega}$ and $n \in \omega$, let $\operatorname{search}(\tau, n)$ be the least $k$ such that:

- there exists a least $\tau_{1} \supseteq \tau$ of length $<k$ such that for all $\tau_{2} \supset \tau_{1}, \mu\left(U^{\tau_{2}}-U^{\tau_{1}}\right)<$ $2^{-2 n-4}$.

- for all $\tau_{0} \supseteq \tau$ less than this $\tau_{1}$ (according to the ordering specified above) there exists $\tau_{2} \supset \tau_{0}$ of length $<k$ with $\mu\left(U^{\tau_{2}}-U^{\tau_{0}}\right) \geq 2^{-2 n-4}$.

In other words $\operatorname{search}(\tau, n)$ is the length of search required in order to extend any leaf $\tau$ of $T$ correctly at stage $n$ in the proof of Theorem 4.2. Now let $f$ be defined by recursion as follows:

$$
\begin{aligned}
f(0) & =\operatorname{search}(\emptyset, 0), \\
f(n+1) & =\max \{\operatorname{search}(\tau, n+1)|| \tau \mid \leq f(n)\} .
\end{aligned}
$$

The point of the function $f$ is that it bounds the length of search necessary to extend $T$ "correctly" at stage $n$ of the construction, even if we have not defined $T$ correctly at previous stages because we did not search for long enough. We take $h \leq_{T} \alpha$ 
which is not dominated by $f$ and at each stage $n$ of the construction we shall now search for $h(n)$ many steps in order to decide how to extend $T$ (rather than $f(n)$ many steps, which is in effect what we did before). The fact that $h(n) \geq f(n)$ for infinitely many $n$ will suffice to show that our approximation to $T^{*}$ converges.

We are now ready to define the construction-we shall explain how we act in order to satisfy (4) and how we use the fact that $h$ is not dominated by $f$ to ensure that our approximation to $T^{*}$ converges as we define the construction.

Stage 0 . Let $\tau$ be the least string such that for all $\tau^{\prime} \supset \tau$ of length $<h(0)$, $\mu\left(U^{\tau^{\prime}}-U^{\tau}\right)<2^{-4}$. Define $T(\emptyset)=T^{*}(\emptyset)=\tau$ and allocate the marker $l_{0}$ to $\tau$.

At stage 0 , then, we proceed exactly as we did in the proof of Theorem 4.2 except that now we only search for $h(0)$ many steps. Clearly (4) is satisfied at the end of stage 0 since there exists just one string which is allocated a marker $l_{0}$ and this string adds less than $2^{-2}$ to $\mu(A)$.

Stage $n>0$. By the end of stage $n-1$ we shall have decided all values $T(\sigma)$ for $\sigma$ of length $\leq n-1$. Since $T$ is partial we may have decided that some of these values should be undefined. We will also have a present approximation to $T^{*}$. For some greatest $m \leq n-1$ we shall have that $T^{*}(\sigma) \downarrow$ for all $\sigma$ of length $\leq m$ and that $T^{*}(\sigma) \uparrow$ for all $\sigma$ of length $>m$. The leaves of $T^{*}$ will be strings of level $n-1$ in $T$. All strings in $T^{*}$ will be allocated a marker (as will some other strings). Assume inductively that (4) is satisfied at the end of stage $n-1$ and that so far we have only allocated markers $l_{n^{\prime}}$ for (some) $n^{\prime} \leq n-1$.

Step (a). The first thing we do at stage $n$ is to form a set of possible candidates for strings of level $n$ in $T$. For each leaf $\tau$ of $T^{*}$ we proceed as follows. For each of the one element extensions of $\tau$, say $\tau_{i}, i=0,1$ we find the least extension $\tau_{i}^{\prime} \supseteq \tau_{i}$ such that for any $\tau^{\prime} \supset \tau_{i}^{\prime}$ of length $<h(n)$ we have $\mu\left(U^{\tau^{\prime}}-U^{\tau_{i}^{\prime}}\right)<2^{-2 n-4}$. We let $C$ be the set of all $\tau_{i}^{\prime}$ chosen in this way (ranging over all leaves $\tau$ ).

Step (b). The second thing we do is to decide which of the strings in $C$ should be enumerated into $T$ and at which level we should extend $T^{*}$. As above, let $m$ be the greatest such that $T^{*}(\sigma) \downarrow$ for all $\sigma$ of length $\leq m$. Our first choice is to extend $T^{*}$ at level $m+1$. This is possible if for every leaf $\tau$ of $T^{*}$ there exist two distinct extensions $\tau^{\prime} \in C$ with $\mu\left(U^{\tau^{\prime}}-U^{\tau}\right)<2^{-2 n-2}$. In this case we enumerate all strings in $C$ into $T$ and into $T^{*}$ (so that these are strings of level $n$ in $T$ and level $m+1$ in $T^{*}$ ). Each of these strings is allocated a marker $l_{n}$. Clearly, in this case, condition (4) is satisfied since there are at most $2^{n}$ strings to which we have just allocated markers $l_{n}$ and each of these strings adds less than $2^{-2 n-2}$ to $\mu(A)$.

Otherwise we must extend $T^{*}$ at a level $m^{\prime} \leq m$ and we do so at the greatest level at which extension is possible. For $\tau \in T$ let $p(\tau)$ be the longest proper initial segment of $\tau$ to which a marker is allocated if there exists such, putting $p(\tau)=\emptyset$ otherwise. Extension is possible at level $m^{\prime}$ if for each $\tau$ of level $m^{\prime}$ in $T^{*}$ there exists an extension, $e(\tau)$ say, in $C$ with $\mu\left(U^{e(\tau)}-U^{p(\tau)}\right)<2^{-2 n^{\prime}-2}$, where $n^{\prime}$ is such that $\tau$ is allocated a marker $l_{n^{\prime}}$. If we extend at level $m^{\prime}$ then for each $\tau=T^{*}(\sigma)$ for $\sigma$ of length $m^{\prime}$ we redefine $T^{*}(\sigma)=e(\tau)$ and enumerate $e(\tau)$ into $T$. We allocate the marker previously allocated to $\tau$ to $e(\tau)$ and remove all markers from strings $\tau^{\prime}$ with $p(\tau) \subset \tau^{\prime} \subset e(\tau)$. We make all values $T^{*}(\sigma)$ for $\sigma$ of length $>m^{\prime}$ undefined. Clearly in this case we have still satisfied (4) since any measure in $A$ can be attributed 
to the same markers as before, except that measure in $U^{e(\tau)}-U^{p(\tau)}$ which can now be attributed to the marker $l_{n^{\prime}}$ on $e(\tau)$-which still contributes less than $2^{-2 n^{\prime}-2}$.

At the very least we shall always be able to extend $T^{*}$ at level 0 . Now suppose that $h(n) \geq f(n)$ and we extend $T^{*}$ at level $m^{\prime}$ at stage $n$. Then each string $\tau$ which we define as a leaf of $T^{*}$ by the end of stage $n$ really will satisfy the property that for all $\tau^{\prime} \supset \tau, \mu\left(U^{\tau^{\prime}}-U^{\tau}\right)<2^{-2 n-4}$. Thus at every stage $>n$ we shall be able to extend $T^{*}$ at a level $>m^{\prime}$.

Verification. It follows immediately by induction that at every stage condition (4) is satisfied, so that the measure of that portion of $A$ already enumerated by the end of stage $n$ is always less than $\frac{1}{2}$. Thus $\mu(A) \leq \frac{1}{2}$.

It remains to show that the approximation to $T^{*}$ converges but this follows immediately from the observation made above, that whenever $h(n) \geq f(n)$ and we extend $T^{*}$ at level $m^{\prime}$ at stage $n$, we shall be able to extend $T^{*}$ at a level $>m^{\prime}$ at every stage $>n$.

$\S 5$. Computably enumerable $L R$ degrees. In this section we study the structure of the c.e. $L R$ degrees and their relationship with the Turing reducibility. The results have been chosen so that they demonstrate how to transfer selected basic techniques from the c.e. Turing degrees (like Sacks coding and restraints) to the c.e. $L R$ degrees. The reader can use these examples in order to prove new results about this structure. We note that the relationship between $\leq_{L R}$ and $\leq_{T}$ is nontrivial and goes beyond what we discuss here. For example there is a half of a minimal pair in the c.e. Turing degrees which is $L R$-complete [1,2]. For background in the theory of c.e. degrees we refer the reader to [21]. The following theorem demonstrates how infinitary Sacks coding can be handled in the $L R$ degrees.

THEOREM 5.1. If $W$ is an incomplete c.e. set, i.e., $\emptyset^{\prime} \not_{T} W$, then (uniformly in $W$ ) there is a c.e. set $B$ such that $B \leq_{L R} W$ and $B \mathbb{Z}_{T} W$.

Proof. A relativisation of the classic non-computable low for random argument of [12] (also see [5]) merely gives that for all $A$ there exists $B$ c.e. in $A$ such that $B \not_{T} A$ and $A \oplus B \leq_{L R} A$. If we assumed that $W$ is low we could prove Theorem 5.1 with a finitary argument similar to [12] by using a lowness technique (namely Robinson's trick). To prove the full result we need infinitary coding combined with cost efficiency considerations (see [14] for examples of cost-function arguments). We need to construct a c.e. operator $V$ and a c.e. set $B$ such that

$$
U^{B} \subseteq V^{W}
$$

where $U$ is a member of the universal oracle Martin-Löf test of lemma 1 (so that $\left.\mu(U)<2^{-1}\right)$,

$$
\mu\left(V^{W}\right)<1
$$

and the following requirements are satisfied

$$
P_{e}: \Phi_{e}^{W}=B \Rightarrow \Gamma_{e}^{W}=\emptyset^{\prime}
$$

where $\left(\Phi_{e}\right)$ is an effective enumeration of all Turing functionals and $\Gamma_{e}$ are Turing functionals constructed by us. It is worth being very clear about the timing of enumerations. We shall suppose that at the beginning of stage $s$ we have already defined $B_{s}$ and that enumerations we make during the course of stage $s$ are in order 
to define $B_{s+1}$. At stage $s$ we let $b_{s}$ be defined as follows: let $n_{s}$ be the least number enumerated into $B$ at stage $s-1$ (i.e., the least number in $B_{s}-B_{s-1}$ ) if there exists such and otherwise let $n_{s}=s$; then $b_{s}=B_{s} \uparrow n_{s}+1$ and $s$ is a true stage for the enumeration of $B$ if $b_{s} \subset B$.

The operator $V$ can be defined ahead of the construction. At the beginning of stage $s$ we enumerate a string $\sigma$ into $V^{W}$ in the following cases:

- there exists $\langle\tau, \sigma\rangle \in U$ such that $\tau \subseteq b_{s}, \sigma$ is not presently in $V^{W}$ and we have not previously enumerated $\sigma$ into $V^{W}$ at any stage $s^{\prime}$ such that $\tau \subseteq b_{s^{\prime}}$. In this case we enumerate $\sigma$ into $V^{W}$ with big (i.e., larger than any number mentioned in the construction so far) $W$-use $u_{W, \tau}(\sigma)$.

- there exists $\langle\tau, \sigma\rangle \in U$ such that $\tau \subseteq b_{s}$, we have previously enumerated $\sigma$ into $V^{W}$ at a stage $s^{\prime}$ such that $\tau \subseteq b_{s^{\prime}}$ but now $\sigma$ is not in $V^{W}$ at the beginning of stage $s$ due to some $W$-change. In this case we enumerate $\sigma$ into $V^{W}$ with the same $W$-use $u_{W, \tau}(\sigma)$ as before.

Note that this definition of $V$ ensures that (7) is satisfied. In the following it is helpful to assume that $V^{W}$ is prefix-free (as a set of finite strings) at all stages. It is clear that we can arrange the enumeration of $V$ so that this holds, while maintaining $U^{B} \subseteq V^{W}$ at all stages. The main conflict we face is that on the one hand we want a Sacks coding for each of the $P_{e}$ requirements (enumerations into $\emptyset^{\prime}$ may trigger $B$-enumerations infinitely often) and on the other hand $B$-enumerations may force $\mu\left(V^{W}\right)=1$ (via the definition of $V$ given above). The connection between $B$-enumerations and superfluous measure in $V^{W}$ (in the sense that it does not serve (7), it corresponds to intervals which are not in $U^{B}$ ) is roughly as in the noncomputable low for random construction of [12]: some interval $\sigma$ is enumerated into $U^{B}$ with use $u$, it enters $V^{W}$ with use $v$ and subsequently $B \uparrow u$ changes thus ejecting $\sigma$ from $U^{B}$. Then $W \uparrow v$ could freeze, thus capturing a useless interval in $V^{W}$. We already have $\mu\left(V^{W}\right) \geq \mu\left(U^{B}\right)$ so we want to make sure that the measure corresponding to useless strings is bounded by $2^{-1}$. Here, however, we have an advantage over the classic argument in [12] as $W$ may also change, thus extracting the useless string from $V^{W}$. We will use this fact in order to make infinitary coding into $B$ possible while satisfying (8). Let

$$
\ell\left(\Phi_{e}^{W}, B\right)[s]=\max \left\{t \mid \Phi_{e}^{W}(n)[s] \downarrow=B(n)[s] \text { for all } n \leq t\right\}
$$

be the length of agreement of the reduction $\Phi_{e}^{W}$ in relation to $B$ at stage $s$.

Idea behind the $P_{e}$-strategy. The functional $\Gamma_{e}$ will be implicit. During the construction we define codes (markers) $k_{n}^{e}$ so that if $k_{n}^{e} \downarrow$ and $n \searrow \emptyset^{\prime}$ then we need to put $k_{n}^{e} \searrow B$. Thus $\emptyset^{\prime}$ changes are coded into $B$ and $B$ changes are coded into $W$ via $\Phi_{e}$ as in the usual Sacks argument: assuming that $\Phi_{e}^{W}=B$ we get $\Gamma_{e}^{W}=\emptyset^{\prime}$, a contradiction. Note that $k_{n}^{e}$ is defined below the length of agreement $\ell\left(\Phi_{e}^{W}, B\right)$; if at some stage this length of agreement drops below $k_{n}^{e}$ then we are allowed to move $k_{n}^{e}$. Our commitment is that if the reduction $\Phi_{e}^{W}=B$ is total (i.e., every marker in this reduction settles down) then every $k_{n}^{e}$ reaches a final position.

Strategy $P_{e}$ can afford cost (i.e., measure of useless strings in $V^{W}$ ) at most $2^{-(e+2)}$. In this way after the construction we can count the measure of $V^{W}$ as $\mu\left(U^{B}\right)$ plus the junk measure for each $P_{e}$ which amounts to a number $<1$ (for such cost counting arguments see [14]). The idea behind the strategy is the following: 
for any $x, s$ we define the absolute cost of $x$ at stage $s$ as

$$
\operatorname{Acost}(x, s)=\mu\left(\sigma \mid \exists\langle\tau, \sigma\rangle \in U \text { with } \tau \subseteq b_{s},|\tau|>x \text { and } u_{W, \tau}(\sigma) \downarrow\right)
$$

and the relative cost of $x$ at stage $s$ as

$$
\operatorname{Rcost}_{e}(x, s)=\mu\left(\sigma \mid \exists\langle\tau, \sigma\rangle \in U \text { with } \tau \subseteq b_{s},|\tau|>x \text { and } u_{W, \tau}(\sigma) \downarrow<v_{e}(x, s)\right)
$$

where $v_{e}(x, s)$ is the use of $\Phi_{e}^{W}(x)[s]$ if this is defined, and $\infty$ otherwise. The absolute cost is the maximum cost we could pay for enumerating $x \searrow B$ and it corresponds to the worst case scenario that there are no helpful $W$-changes (as in [12] where $W$ is empty). Note that we would not enumerate a code $x$ into $B$ unless $\ell\left(\Phi_{e}^{W}, B\right)>x$. If this diagonalization is unsuccessful then $W\lceil v(x, s)$ will change. The relative cost is the expected cost under the assumption that the $\Phi_{e}$-computation will recover.

It is important to note that during our attempts to achieve $\Phi_{e}^{W} \neq B$ we will have to pay the absolute cost at most once but we might have to pay relative costs infinitely often (due to the infinitary coding). So we split the amount $2^{-(e+2)}$ allowed into two and we are going to restrict the absolute cost to $2^{-(e+3)}$ and the total of relative costs to $2^{-(e+3)}$. Now we split the relative cost allowance to the markers $k_{n}^{e}$ that the strategy operates: the enumeration of $k_{n}^{e}$ should not cost more than $2^{-(e+n+4)}$ (unless $W$ does not respond to this diagonalization, in which case we can count the cost of $k_{n}^{e}$ as the absolute cost). Notice that $k_{n}^{e}$ (whatever values it takes in the course of the construction) will be enumerated into $B$ at most once (so it will cost us at most once) since we only enumerate it into $B$ if $n \searrow \emptyset^{\prime}$.

If the strategies respect the above quotas when they perform their coding ( $B$ enumerations) then (8) is satisfied (by a standard cost-counting argument relative to $W$ ). Now we only need to show that the Sacks coding can work under the above cost efficiency restrictions. This will follow from the following lemma, which will be proved once we have defined the construction.

Lemma 5.2. If $\Phi_{e}^{W}=B$ is total then all markers $k_{n}^{e}$ such that $n \notin \emptyset^{\prime}$ will be permanently defined (despite the tough cost-related conditions).

The proof of this lemma is just a slight extension of the standard cost function arguments that $\lim _{x} \sup _{t} \operatorname{cost}(x, t)=0($ see $[12,14])$. Based on the assumption that Lemma 5.2 holds $P_{e}$ will place the next marker $k_{n}^{e}$ onto a number $x_{0}$ such that $\operatorname{Rcost}\left(x_{0}, s\right)$ is currently less than the relative cost allowance $q_{n}$ for $k_{n}^{e}$. Then according to the definition of $V$, in later stages $R \operatorname{cost}\left(x_{0}\right)$ will remain less than $q_{n}$ (as new measure in $V^{W}$ comes with big $W$-use), although the absolute cost may increase. Thus the coding can go through by keeping the relative cost below quota.

There are some final points that we need to sort out in order to ensure that $P_{e}$ can perform the coding under the cost efficiency restrictions. Although we managed to keep the relative cost below quota, the absolute cost of enumerating some $k_{n}^{e} \downarrow$ into $B$ may rise above the threshold $2^{-(e+3)}$ after we define $k_{n}^{e}$. In fact, 'false' measure in $U^{b_{s}}$ (i.e., strings $\sigma$ in $U^{b_{s}}$ which are not in the final value $U^{B}$ ) may mean that this happens for an infinite number of markers at an infinite number of stages of the construction. The solution is that, once $n \searrow \emptyset^{\prime}$, the marker $k_{n}^{e}$ will have to wait for a stage at which the absolute cost drops below quota before being enumerated into $B$. Since at every true stage in the enumeration of $B$ we have that $U^{b_{s}}$ is actually 
a subset of the final value $U^{B}$, for all but a finite number of markers the cost will drop below quota at all true stages of the construction.

When we define a marker $k_{n}^{e}$ we shall choose this value from $\mathbb{N}[\langle e, n\rangle]$. In order to ensure that Lemma 5.2 holds we shall allow that when a marker is defined below the length of agreement we may redefine this value to be less than its previous value. Clearly this poses no problems in the satisfaction of $P_{e}$. When we define a value $k_{n}^{e}$ the value $\tau\left(k_{n}^{e}\right)$ will be the initial segment of $W_{s}$ below the use of the computation $\Phi_{e}^{W}\left(k_{n}^{e}\right)$. Now we state the actual $P_{e}$-strategy and the construction. A stage $s$ is called expansionary if $\ell\left(\Phi_{e}^{W}, B\right)[s]>\ell\left(\Phi_{e}^{W}, B\right)[t]$ for all $t<s$.

$P_{e}$-strategy. At stage $s$ let $\ell\left(\Phi_{e}^{W}, B\right)$ be the current length of agreement and do the following:

1. For each $n \notin \emptyset_{s}^{\prime}$ with $k_{n}^{e} \downarrow$ for which there exists $x<k_{n}^{e}$ in $\mathbb{N}^{[\langle e, n\rangle]}$ with $R \operatorname{cost}(x, s)<2^{-(e+n+4)}$ redefine $k_{n}^{e}:=x$ and define $\tau\left(k_{n}^{e}\right)$ to be the initial segment of $W_{s}$ below the use in the computation $\Phi_{e}^{W}(x)$.

2. For each $k_{n}^{e}$ such that $\tau\left(k_{n}^{e}\right) \nsubseteq W_{s}$ make $k_{n}^{e} \uparrow$.

3. Look for the least $n$ such that $n \notin \emptyset_{s}^{\prime}, k_{n}^{e} \uparrow$ and check if there is $x<\ell\left(\Phi_{e}^{W}, B\right)$, $x \in \mathbb{N}[\langle e, n\rangle]$ such that $R \operatorname{cost}(x, s)<2^{-(e+n+4)}$. If not do nothing, otherwise define $k_{n}^{e}=x$ for the least such $x$ and define $\tau\left(k_{n}^{e}\right)$ to be the initial segment of $W_{s}$ below the use in the computation $\Phi_{e}^{W}(x)$. If $P_{e}$ has not had an expansionary stage subsequent to the last stage at which it made an enumeration then do nothing more. Otherwise (or if the strategy has not previously enumerated any markers into $B$ ) go to (4).

4. For the least $n \in \emptyset_{s}^{\prime}$ such that $k_{n}^{e} \downarrow \notin B$ and $A \operatorname{cost}\left(k_{n}^{e}, s\right)<2^{-(e+3)}$, put $k_{n}^{e} \searrow B$ (if there exists such).

Construction. At stage $s$ we access strategies $P_{i}, i<s$ and let them act through their steps.

Verification. First we show that (8) is satisfied. Figure 1 illustrates how strings go in and out of the sets $V^{W}[s], U^{B}[s]$ and $V^{W}[s]-U^{B}$ during the stages $s$ of the construction. The set $V^{W}$ consists of the strings which have entered $V^{W}$ with correct $W$-use. This collection of strings can be divided into two categories: the ones which came through a $B$-correct enumeration (as in the definition of the $V^{W}$ enumeration) and the ones which did not. The first category of strings has measure $\mu\left(U^{B}\right)<2^{-1}$. The second category strings are the ones which enter the $P_{e}$ cells of Figure 1: every time a first category string moves to second category during the construction it means that this string was in $U^{B}$ and was then extracted due to a $B$-change. Such a $B$-enumeration must have happened in a particular substage by a particular strategy $P_{e}$. Hence this string goes to the $P_{e}$ cell.

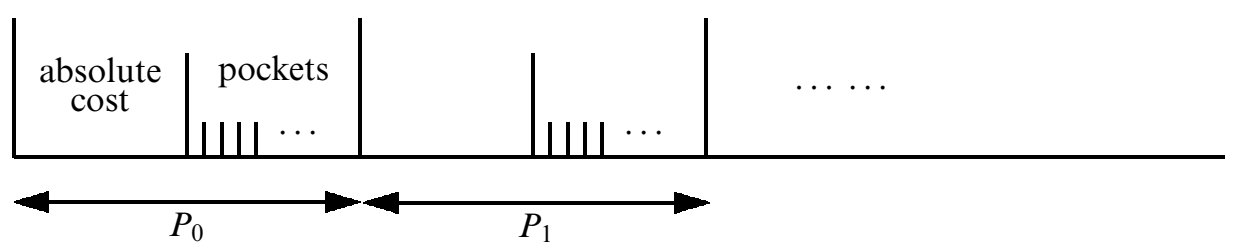

FIGURE 1. Junk measure distribution during the construction. 
Each $P_{e}$ cell is divided into two main compartments: the first compartment corresponds to the absolute cost and the second to the relative costs. Every time a string goes to the $P_{e}$ cell (as explained above) it first goes to the first compartment (which may contain many strings but all of them must have come from a single $B$ change). Note that this load is associated with a particular marker $k_{n}^{e}$ of $P_{e}$ which caused the $B$-change. The second compartment is divided into infinitely many pockets corresponding to the markers $k_{n}^{e}, n \in \mathbb{N}$. If and when $P_{e}$ goes through a later $\Phi_{e}$-expansionary stage, part of the load exits $V^{W}$ (due to the associated $W$ change) and part of it stays in $V^{W}$. During such an expansionary stage the remaining load has measure at most the relative cost of $k_{n}^{e}$ and is emptied in the $k_{n}^{e}$-pocket of the second compartment.

By the cost quotas that we respected in the construction the measure of the eventual load of the $P_{e}$ cell will be less than $2^{-(e+2)}$. Indeed, the first compartment of $P_{e}$ will eventually measure less than the absolute cost quota $2^{-(e+3)}$ and the second compartment has to measure less than the sum of all relative costs $\sum_{n} 2^{-(e+n+4)}=$ $2^{-(e+3)}$. Hence the measure of the strings of the second category is less than $\sum_{e} 2^{-(e+2)}=2^{-1}$. This shows that $\mu\left(V^{W}\right)<1$.

Next we prove Lemma 5.2. So suppose towards a contradiction that $\Phi_{e}^{W}=B$ and $n$ is the least such that $n \notin \emptyset^{\prime}$ and there is no stage of the construction after which $k_{n}^{e}$ is always defined to take the same value. Let $x \in \mathbb{N}^{[\langle e, n\rangle]}$ be such that $\mu\left(U^{B}\right)-\mu\left(U^{B \nmid x}\right)<2^{-(e+n+4)}$. Let $s_{0}$ be large enough such that $B_{s_{0}} \uparrow x+1 \subset B$, $\ell\left(\Phi_{e}^{W}, B\right)>x+1$ at stage $s_{0}$ and $W_{s_{0}}\left\lceil v_{e}\left(x, s_{0}\right) \subset W\right.$. Let $s_{1}>s_{0}$ be a true stage in the enumeration of $B$. Then the marker $k_{n}^{e}$ will be defined to take the same value at all stages $\geq s_{1}$ : if it is already defined it will move to a position $\leq x$ through step (1); otherwise it will be newly defined to a value $\leq x$; in any case it will be permanently defined because $s_{1}$ is a true stage. This gives the required contradiction.

Finally it is now straightforward to argue that all $P_{e}$ are satisfied. Suppose that $\Phi_{e}^{W}=B$. Let $m$ be large enough such that for all $n \geq m$ with $n \notin \emptyset^{\prime}$, $\mu\left(U^{B}\right)-\mu\left(U^{B}\left\lceil k_{n}^{e}\right)<2^{-(e+3)}\right.$ (letting $k_{n}^{e}$ take its final value). In order to compute $\emptyset^{\prime}(n)$ for any $n \geq m$ using an oracle for $W$, run the construction until a stage $s$ is found such that either $n \in \emptyset_{s}^{\prime}$ or $k_{n}^{e}$ is defined and below the length of agreement with a $W$-correct computation. We have that $n \in \emptyset^{\prime}$ iff it has been enumerated by stage $s$.

It is easy to see that the above proof works even if we require $U^{B \oplus W} \subseteq V^{W}$ instead of (7). In that case we obtain $B \oplus W \equiv_{L R} W, W<_{T} B \oplus W$ and hence the following theorem, given that there are $T$-incomplete sets in the complete $L R$-degree and the known embedding results for the c.e. Turing degrees (an antichain is embeddable in every nontrivial interval).

THEOREM 5.3. Every c.e. LR degree contains infinitely many c.e. Turing degrees (in the form of chains and antichains) and the only T-topped (i.e., containing a maximum/maximal $T$-degree) c.e. LR degree is the complete $L R$ degree.

As far as the global structure is concerned, we can get a similar result by relativising known constructions of low for random degrees. In particular, the relativised noncomputable low for random construction [12] gives that for every $B$ there is $A$ which is $B$-c.e. and $A \oplus B \equiv_{L R} B, B<_{T} A \oplus B$; and a slight extension of 
the argument gives that every $B$ is $T$-below an antichain of $T$-degrees in the same $L R$-degree, hence the following theorem.

THeOREM 5.4. Every LR degree contains infinitely many Turing degrees (in the form of chains and antichains) and no maximal Turing degree.

Next we show a splitting theorem which also shows how Sacks restraints work in the $L R$ degrees.

THEOREM 5.5. If $A$ is c.e. and not low for random then there are c.e. $B, C$ such that

- $B \cap C=\emptyset$,

- $B \cup C=A$,

- $A \underline{L}_{L R} B$ and $A \not L_{L R} C$.

Proof. The main idea is as in the classic Sacks splitting theorem. We just have to translate the main tools like length of agreement and Sacks restraints to the case of $L R$ reductions. This will not be a problem as $\leq_{L R}$ is $\Sigma_{3}^{0}$. Fix a member $U$ of a universal oracle Martin-Löf test; an $L R$ reduction is defined via a c.e. operator $V$ (as opposed to a Turing functional) and a $q \in \mathbb{Q}_{2}, 0<q<1\left(\mathbb{Q}_{2}\right.$ are the rationals with finite binary expansion) such that $\mu\left(V^{\beta}\right)<q$ for all $\beta \in 2^{\omega}$ (it is easy to see that there is an effective enumeration of all such pairs $V, q)$. Then $A$ is $L R$ reducible to $B$ via $V, q$ if

$$
U^{A} \subseteq V^{B}
$$

To define the length of agreement $\ell\left(U^{A}, V^{B}\right)$ of this possible reduction consider computable enumerations of $U, V, A, B$. Let $\left(\sigma_{s}\right)$ be a recursive enumeration of all the finite strings which appear in $U_{t}^{A_{t}}$ at some stage $t$, and such that each $\sigma$ appears once in this list for each time that it appears in $U_{t}^{A_{t}}$ with new use. If $\sigma_{s}$ is enumerated into this list at stage $t$, then at stage $t^{\prime}>t$ we say that $\sigma_{s}$ has remained in $U^{A}$ if it is in $U_{t^{\prime}}^{A_{t^{\prime}}}$ with the same use. Now for all $s$ we define $\ell\left(U^{A}, V^{B}\right)[s]$ to be the maximum $n$ such that the following hold:

- $\sigma_{n}[s] \downarrow$ (that is, the $n$th member of this sequence has been enumerated by stage $s$ ),

- $\forall i \leq n\left(\left[\sigma_{i}\right] \subseteq V_{s}^{B_{s}} \vee \sigma_{i}\right.$ has not remained in $\left.U^{A}\right)$.

Assuming the standard hat-trick for the $\Sigma_{2}^{0}$ approximation of $U^{A}$ (i.e., let $k_{s}=$ $\min \{x: x \in A[s]-A[s-1]\}$, or $k=s$ if there are no such $x$ and define $\widehat{A}[s]=$ $A[s] \uparrow k$; then (re)define $U^{A}[s]:=\left\{\sigma:\langle\sigma, \tau\rangle \in U_{s}\right.$ for some $\left.\tau \subseteq \widehat{A}[s]\right\}$ ) it is clear that reduction (9) is correct iff

$$
\liminf _{s} \ell\left(U^{A}, V^{B}\right)[s]=\infty .
$$

Now in general, if we wish to destroy a given reduction like (9) where $A$ is a given c.e. set of nontrivial $L R$ degree and $B$ is enumerated by us, it's enough if we respect the following restraint at every stage $s$ :

$$
r(V, q, s)=\mu t\left[\forall i \leq \ell\left(U^{A}, V^{B}\right)[s]\left(\left[\sigma_{i}\right] \subseteq V_{s}^{B_{s}} \text { with } B \text {-use }<t \vee \sigma_{i} \notin U_{s}^{A_{s}}\right)\right]
$$

Indeed, we claim that if $r(V, q, s)$ is respected for a cofinite set of stages then

$$
\lim _{s} \ell\left(U^{A}, V^{B}\right)[s]<\infty .
$$


To show this suppose after stage $s_{0}$ the restraint is respected and start enumerating a set of strings $E$ as follows: at stage $s$ put $\sigma_{i} \searrow E$ if $i \leq \ell\left(U^{A}, V^{B}\right)[s]$ and $\sigma_{i} \in U_{s}^{A_{s}}$. It is clear that after $s_{0}, \ell\left(U^{A}, V^{B}\right)$ does not decrease. Thus, if (10) did not hold, $U^{A} \subseteq E$. Also, $\mu(E)<1-q$ by the properties of $V$ and since $E \subseteq V^{B}$ by the restraints on $B$. But this contradicts the assumption that $A \not_{L R} \emptyset$, given that $E$ is computably enumerable.

So there must be some $i$ such that $\sigma_{i}$ is a permanent resident of $U^{A}$ and $\sigma_{i}$ is never covered by strings in $V^{B}$. This means that (9) is destroyed and the restraint comes to a limit. This is all we needed in order to apply the classic Sacks splitting argument. Consider an effective enumeration $\left(V_{e}, q_{e}\right)$ of all tuples $(V, q)$ where $V$ is a c.e. operator (for all $\beta \in 2^{\omega}, V^{\beta} \subseteq 2^{<\omega}$ ) and $q \in \mathbb{Q}_{2} \cap(0,1)$ with $\mu\left(V^{\beta}\right)<1-q$ for all $\beta \in 2^{\omega}$. To prove the theorem we need to enumerate $B, C$ such that $A=B \cup C$, $B \cap C=\emptyset$ and the following requirements are satisfied:

$$
\begin{aligned}
& R_{e}: U^{A} \nsubseteq V_{e}^{B}, \\
& Q_{e}: U^{A} \nsubseteq V_{e}^{C} .
\end{aligned}
$$

The rest of the proof is as in the Sacks splitting theorem (see [21] for a presentation), only that the length of agreement and the restraints are defined as above.

§6. Digression: Enumerations of random sets. It is a classical result of computability theory that every level of the arithmetical hierarchy is proper i.e., that for every $n \geq 1$ there exist sets which are $\Sigma_{n}^{0}$ and which are not $\Delta_{n}^{0}$. Of course it is also well known (see [6,17]) that for every $n \geq 1$ there exist sets which are $n$-c.e. and which are not $(n-1)$-c.e. (by 0 -c.e. we mean computable). These are called properly $n$-c.e. sets. It seems a natural question, therefore, to ask what can be said along these lines where we restrict ourselves to the case of random sets.

THeOREm 6.1. For every $m, n \geq 1$ there exist sets which are $n$-random and which are properly $m$-c.e. in $\emptyset^{(n)}$.

The easiest way to show this is via the following theorem of Joe Miller (currently available in [14]). Let $\mathscr{P}$ be any $\Pi_{1}^{0}$ class and $A \in \mathscr{P}$; we say that $I$ is an independent set for $A$ in $\mathscr{P}$ if whenever $B$ satisfies the property that for all $n \notin I, B(n)=A(n)$ we have $B \in \mathscr{P}$. Also, a set $A$ is called autoreducible (see [22]) if for each $x$ the question 'is $x$ in $A$ ?' can be answered recursively in $A$, without ever asking the oracle about $x$.

Theorem 6.2 (Joe Miller). Suppose that $A \in \mathscr{P}$ is not autoreducible. Then there exists an infinite set I which is an independent set for $A$ in $\mathscr{P}$ and which is computable in $A^{\prime}$. (Also, no random set is autoreducible.)

To show Theorem 6.1 recall that there exists a $\Pi_{1}^{0}$ class $\mathscr{P}$ consisting only of random sets. Apply the low basis theorem in order to obtain a random $A$ and also $I$ which is an independent set for $A$ in $\mathscr{P}$, computable in $\emptyset^{\prime}$. The theorem then follows simply by carrying out the standard construction of a properly $n$-c.e. set within $I$ in place of the identity tree and by relativizing. In the same way, by the argument that produces properly $n$-c.e. degrees for every $n \geq 1$ (see [3,7]), one can see that Theorem 6.1 also holds degree-theoretically: for every $m, n \geq 1$ there exist sets which are $n$-random, $m$-c.e. in $\emptyset^{(n)}$ and Turing equivalent to no $(m-1)$-c.e. in $\emptyset^{(n)}$ set. 
§7. Low for random are $\Delta_{2}^{0}$ revisited. These days the fact that low for random sets are $\Delta_{2}^{0}$ is proved via the fact that this class coincides with the $K$-trivial reals [16] (given that it is much easier to show that $K$-trivials are $\Delta_{2}^{0}$, see [5, 14] for a presentation). However this was originally proved by Nies [15] who showed that for any low for random real there exists a $\Pi_{1}^{0}$ class relative to $\emptyset^{\prime}$ which contains it and has finitely many elements. That proof involved prefix-free complexity and in particular, it constructed a prefix-free machine with certain properties. Below we adapt his argument to our framework; the main advantage is that we only have to deal with natural $\Pi_{1}^{0}$ classes relative to $\emptyset^{\prime}$ which stem from the definition of an oracle MartinLöf test. Notice that in the proof of Lemma 2.1 we first constructed an oracle Martin-Löf test $\left(U_{e}\right)$ with the property

For every oracle Martin-Löf test $\left(V_{e}\right)$, uniformly on its c.e. index we can compute $k \in \mathbb{N}$ such that $V_{e+k} \subseteq U_{e}$ (as sets of axioms) for all $e$.

and then we modified it in order to satisfy the other conditions.

THEOREM 7.1. If $U$ is a member of an oracle Martin-Löf test satisfying condition (11) and $T \in \Sigma_{1}^{0}, \mu(T)<1$ then there are only finitely many $\beta \in 2^{\omega}$ with the property $U^{\beta} \subseteq T$.

Proof. It is enough to find some $c \in \mathbb{N}$ such that in every prefix-free set of strings of cardinality $c$ there is a string $\sigma$ such that $U^{\sigma} \nsubseteq \mathbb{T}$. We will obtain $c$ as a fixed point of our construction. Let $f$ be a computable function such that for every c.e. index $e$ of an oracle Martin-Löf test $\left(V_{i}\right), f(e)$ is such that $V_{f(e)} \subseteq U$. Effectively in given $k$ we construct a c.e. operator $V$ such that for all $\beta \in 2^{\omega}, \mu\left(V^{\beta}\right)<2^{-f(k)}$. Clearly, given an index for such $V$ (and $k$ ) we can effectively get an index of an oracle Martin-Löf test whose $f(k)$-th member is $V$. So by the recursion theorem there is $k$ such that the corresponding $V$ is the $f(k)$-th member of the oracle Martin-Löf test with index $k$. Hence, $V \subseteq U$. We build this $V$ such that there are only finitely many $\beta \in 2^{\omega}$ with $V^{\beta} \in T$.

Our strategies $\alpha$ run over all prefix-free collections of strings of cardinality $c=$ $2^{f(k)+1}$. Let $q>0$ be a rational such that $\mu(T)<1-q$ and $\left(n_{\alpha}\right)$ an effective coding of the strategies into $\mathbb{N}$. Strategy $\alpha$ will use intervals of measure

$$
p_{\alpha}=q \cdot 2^{-n_{\alpha}} \cdot 2^{-1} \text {. }
$$

Construction of $V$. At stage $s$ consider those strategies $\alpha$ which require attention and let them act. A strategy $\alpha$ requires attention at stage $s$ if for all $\tau \in \alpha$, $V^{\tau}[m+1] \subseteq T_{s}$ where $m$ is the last stage that $\alpha$ acted (and 0 otherwise). Also, a strategy $\alpha$ acts as follows. $M_{s}$ is a finite set of strings which covers the reals which are used by stage $s$. We start with $M_{0}=\emptyset$. The strategy chooses $|\alpha|$ finite sets of strings $C_{i} \subseteq \bar{T}$ from $\bar{T}-M_{s-1}$ which are mutually disjoint (as $\Sigma_{1}^{0}$ classes) and have $\mu\left(C_{i}\right)=p_{\alpha} \cdot \frac{1}{c}$. Then it enumerates them into $V^{\alpha_{i}}$ respectively (where $\alpha_{i}$ is the $i$ th member of $\alpha$ ) and into $M_{s}$.

Verification. By induction and the choice of $p(\alpha)$ it is easy to see that when a strategy requires attention it can find the required sets $C_{i}$. Indeed, call $\alpha$ pending at $t$ if it does not require attention at $t$ and let $P_{t}$ be the set of these strategies. Then 
$\mu\left(M_{t}\right)$ is the measure reserved by strategies at $t$ (i.e., the measure enumerated in $\left.\cup_{\rho} V_{t}^{\rho}\right)$ and

$$
\mu\left(M_{t} \cap \overline{T_{t}}\right)<\sum_{\alpha \in P_{t}} p_{\alpha}
$$

since strategy $\alpha$ can only reserve measure $p_{\alpha}$ at a time, and it does not reserve more clopen sets until the previous ones have appeared in $T$. Hence

$$
\mu\left(\overline{T_{t}}-M_{t} \cap \overline{T_{t}}\right)>q-\sum_{\alpha \in P_{t}} p_{\alpha}
$$

which shows the claim, given that $\sum_{\alpha} p_{\alpha}<q$. Also, it can be easily shown that if $P$ is the complement of a finite collection of strings then it can be covered by a finite collection of strings and so, for any dyadic rational $\varepsilon<\mu(P)$ we can effectively find a finite set $C$ such that $\mu(C)=\varepsilon$ and $C \subseteq P$.

It is easily seen that for every $k$ the construction corresponding to $k$ gives $V$ such that for every $\beta \in 2^{\omega}, V^{\beta}<2^{-f(k)}$, since whenever a strategy $\alpha$ adds measure to $V^{\rho}$ for $\rho \subset \beta$ it also adds $2^{f(k)}$ times that measure to $V^{\rho^{\prime}}$ for $\rho^{\prime}$ which are incompatible with $\rho$-and it is clear that whenever $\rho$ and $\rho^{\prime}$ are incompatible we have $V^{\rho} \cap V^{\rho^{\prime}}=\emptyset$.

Finally we show that if $k$ is the fixed point mentioned above (so that $V \subseteq U$ ), then there do not exist $c$ incomparable strings $\rho$ such that $U^{\rho} \subseteq T$. Indeed, otherwise the corresponding strategy $\alpha$ would act at an infinite number of stages putting $p_{\alpha}$ measure into $T$ each time, a contradiction.

Note that if $V$ is a member of an oracle Martin-Löf test and $T \in \Sigma_{1}^{0}$ then the class $\left\{\beta \mid V^{\beta} \subseteq T\right\}$ is $\Pi_{1}^{0}$ relative to $\emptyset^{\prime}$, i.e., it consists of the infinite paths through a $\boldsymbol{O}^{\prime}$ computable tree. Since the paths through a $\boldsymbol{O}^{\prime}$ computable tree with only finitely many infinite paths are $\Delta_{2}^{0}$ by Theorem 3.2 we get Nies' result [15] that all low for random sets are $\Delta_{2}^{0}$. However we wish to note that there are members $V$ of universal oracle Martin-Löf tests for which the property of Theorem 7.1 does not hold, i.e., there are infinitely many $\beta$ with $V^{\beta} \subseteq T$.

To construct such a test one first fixes a uniformly computable infinite family of reals $\left(\beta_{i}\right)$. We then proceed just as we did in the proof of Lemma 2.1 in order to define the second element of that test, only that now we avoid enumerating axioms $\langle\tau, \sigma\rangle$ such that $\tau \neq \emptyset, \tau \subset \beta_{i}$. This is done by replacing this axiom with axioms $\left\langle\tau^{\prime}, \sigma\right\rangle$ where $\tau^{\prime} \supset \tau$ and $\tau^{\prime} \nsubseteq \beta_{i}$ for all $i$. Fixing the second element of some universal (non-oracle) test $U^{\prime}$ we also enumerate all axioms $\langle\emptyset, \sigma\rangle$ such that $\sigma \in U^{\prime}$. Note that the only reals $\beta$ such that $V^{\beta}$ loses reals (in comparison with the original test) are the ones in the family $\left(\beta_{i}\right)$. But this is no harm as for computable $\beta$, the $\beta$-random reals are the $\emptyset$-random reals. However by Theorem 3.2 and the fact that the class of low for random reals is countable, if $V$ is a member of a universal oracle Martin-Löf test there can only be countably many $\beta$ such that $V^{\beta} \subseteq T$.

\section{REFERENCES}

[1] George Barmpalias and Antonio Montalban, A cappable almost everywhere dominating computably enumerable degree, Electronic Notes in Theoretical Computer Science, vol. 167 (2007), pp. 17-31. 
[2] Stephen Binns, BJørn Kuos-Hanssen, Joseph S. Miller, and Reed Solomon, Lowness notions, measure and domination, in preparation.

[3] S. BARry COOPER, Computability Theory, Chapman \& Hall/ CRC Press, Boca Raton, FL, New Yo ondon, 2004.

[4] Natasha L. Dobrinen and Stephen G. Simpson, Almost everywhere domination, this Journal, vol. 69 (2004), no. 3, pp. 914-922.

[5] Rodney Downey and Denis Hirschfeldt, Algorithmic Randomness and Complexity, Springer, 2008 , in print. Current draft available at http://www.mcs . vuw.ac.nz/ downey/.

[6] Yu. L. Ershov, A hierarchy of sets, Algebra i Logika, vol. 7 (1968), pp. 47-74, translation: Algebra and Logic, vol. 7 (1968), pp. 25-43.

[7] L. HAY and M. LeRMAN, On the degrees of boolean combinations of r.e. sets, Recursive Function Theory Newsletter, 1976.

[8] CARL G. Jockusch, Simple proofs of some theorems on high degrees of unsolvability, Canadian Journal of Mathematics, vol. 29 (1977), no. 5, pp. 1072-1080.

[9] BJøRn KJos-HansSEn, Low for random reals and positive-measure domination, Proceedings of the American Mathematical Society, vol. 135 (2007), pp. 3703-3709.

[10] STUART A. KURTZ, Randomness and genericity in the degrees of unsolvability, Ph.D. thesis, University of Illinois at Urbana-Champaign, 1981.

[11] ANTONín KuČERA, Measure, $\Pi_{1}^{0}$ classes and complete extensions of PA, Recursion Theory Week (Oberwolfach, 1984), Lecture Notes in Mathematics, vol. 1141, Springer, Berlin, 1985, pp. 245-259.

[12] Antonín KuČera and Sebastiaan A. Terwijn, Lowness for the class of random sets, this JourNAL, vol. 64 (1999), pp. 1396-1402.

[13] Manuel Lerman, Degrees of Unsolvability: Local and Global Theory, Springer-Verlag, 1983.

[14] ANDRÉ NIES, Computability and Randomness, monograph to appear. Current draft available at http://www.cs. auckland.ac.nz/ nies/.

[15] - Low for random sets: The story, unpublished draft, which is available at the author's webpage http://www.cs . auckland.ac.nz/ nies/. 305.

[16] _ Lowness properties and randomness, Advances in Mathematics, vol. 197 (2005), pp. 274

[17] Piergiorgio Odifreddi, Classical Recursion Theory, vol. I and II, North-Holland, Amsterdam, Oxford, 1989 and 1999.

[18] Gerald Sacks, Degrees of Unsolvability, Princeton University Press, 1963.

[19] Richard Shore and TheOdore Slaman, Working below a high recursively enumerable degree, this JOURNAL, vol. 58 (1993), pp. 824-859.

[20] Stephen G. Simpson, Almost everywhere domination and superhighness, Mathematical Logic Quarterly, vol. 53 (2007), pp. 462-482.

[21] Robert I. Soare, Recursively Eumerable Sets and Degrees, Springer-Verlag, Berlin, London, 1987.

[22] B. A. Trakhtenbrot, On autoreducibility, Rossiskaya Akademiya Nauk, vol. 192 (1970), pp. 1224-1227, translation: Soviet Mathematics Doklady, vol. 11 (1970), pp. 814-817.

\author{
UNIVERSITY OF LEEDS \\ SCHOOL OF MATHEMATICS \\ LEEDS. LS2 9JT, UK \\ E-mail: georgeb@maths.leeds.ac.uk \\ UNIVERSITA DEGLI STUDI DI SIENA \\ DIPARTIMENTO DI SCIENZE MATEMATICHE ED INFORMATICHE \\ VIA DEL CAPITANO 15, 53100 SIENA. ITALY \\ E-mail: andy@aemlewis.co.uk \\ UNIVERSITY OF LEEDS \\ SCHOOL OF MATHEMATICS \\ LEEDS, LS2 9JT, UK \\ E-mail:mariya@maths.leeds.ac.uk
}

